\title{
Superintegrable Stäckel Systems on the Plane: Elliptic and Parabolic Coordinates ${ }^{\star}$
}

\author{
Andrey V. TSIGANOV
}

\author{
St. Petersburg State University, St. Petersburg, Russia \\ E-mail: andrey.tsiganov@gmail.com
}

Received April 10, 2012, in final form May 21, 2012; Published online May 25, 2012

http://dx.doi.org/10.3842/SIGMA.2012.031

\begin{abstract}
Recently we proposed a generic construction of the additional integrals of motion for the Stäckel systems applying addition theorems to the angle variables. In this note we show some trivial examples associated with angle variables for elliptic and parabolic coordinate systems on the plane.
\end{abstract}

Key words: integrability; superintegrability; separation of variables; Abel equations; addition theorems

2010 Mathematics Subject Classification: 37J35; 70H06

\section{Introduction}

In classical mechanics Hamiltonian system on a $2 n$-dimensional phase space $M$ is called completely integrable in Liouville's sense if it possesses $n$ functionally independent integrals of motion $H_{1}, \ldots, H_{n}$ in involution:

$$
\frac{d H_{i}}{d t}=\left\{H, H_{i}\right\}=0, \quad\left\{H_{i}, H_{j}\right\}=0, \quad i, j=1, \ldots, n,
$$

where $H=H_{1}$ is the Hamilton function and $\{\cdot, \cdot\}$ is the Poisson bracket on $M$.

Superintegrable system is a system that is integrable in the Liouville sense and that possesses more functionally independent integrals of motion than degrees of freedom. The construction of superintegrable Stäckel systems using angle variables $\omega_{k}$ has been proposed in $[12,13,14,15]$.

In generic case the action variables $\omega_{k}$ are multi-valued functions on the whole phase space $M$. In fact, we can extract polynomial integrals of motion from angle variables only when we can apply addition theorems to the corresponding Abelian integrals. As there are only few addition theorems for the Abel equations [1,3] we can easily classify the corresponding superintegrable systems, see $[12,13,14,15]$.

The goal of this brief note is to present some trivial examples of applying this generic theory associated with elliptic and parabolic coordinate systems on the plane. Superintegrable systems separable in spherical coordinates can be found in $[2,6]$. The corresponding addition integrals of motion are related with an addition theorem for the logarithmic angle variables. Of course, there is a trivial generalization of the proposed method for all the orthogonal coordinate systems in $\mathbb{R}^{3}$ (ellipsoidal, paraboloidal, cylindrical, prolate and oblate spheroidal coordinates etc).

The non-Stäckel superintegrable systems in classical and quantum mechanics have been considered in $[7,9]$. In contrast with the Stäckel case we do not have a generic theory for constructing such superintegrable systems.

${ }^{\star}$ This paper is a contribution to the Special Issue "Superintegrability, Exact Solvability, and Special Functions". The full collection is available at http://www.emis.de/journals/SIGMA/SESSF2012.html 


\section{The Stäckel systems}

The system associated with the name of Stäckel [11] is a holonomic system on the phase space $\mathbb{R}^{2 n}$, with the canonical variables $q=\left(q_{1}, \ldots, q_{n}\right)$ and $p=\left(p_{1}, \ldots, p_{n}\right)$ :

$$
\Omega=\sum_{j=1}^{n} d p_{j} \wedge d q_{j}, \quad\left\{p_{j}, q_{k}\right\}=\delta_{j k}
$$

The nondegenerate $n \times n$ Stäckel matrix $S$, whose $j$ column depends only on coordinate $q_{j}$, defines $n$ functionally independent integrals of motion

$$
H_{k}=\sum_{j=1}^{n}\left(S^{-1}\right)_{j k}\left(p_{j}^{2}+V_{j}\left(q_{j}\right)\right)
$$

the separated relations

$$
p_{j}^{2}=\sum_{k=1}^{n} H_{k} S_{k j}\left(q_{j}\right)-V_{j}\left(q_{j}\right)
$$

and the action variables $w_{k}$

$$
\omega_{i}=\frac{1}{n} \sum_{j=1}^{n} \int^{q_{j}} \frac{S_{i j}(\lambda)}{\sqrt{\sum_{k=1}^{n} H_{k} S_{k j}(\lambda)-V_{j}(\lambda)}} \mathrm{d} \lambda,
$$

so that

$$
\left\{H_{j}, H_{k}\right\}=\left\{\omega_{i}, \omega_{k}\right\}=0, \quad\left\{H_{j}, \omega_{k}\right\}=\delta_{i j} .
$$

In generic case the action variables (2.1) are sums of the multi-valued Abelian integrals. However, if we are able to apply the known addition theorems for these Abelian integrals then we can get additional integrals of motion [12, 13, 14].

Let us discuss addition theorems for the logarithmic (exponential) and elliptic functions [1, 3$]$. In the first case polynomials

$$
P_{j}=\sum_{k=1}^{n} H_{k} S_{k j}(\lambda)-V_{j}(\lambda)
$$

are the second-order polynomials and

$$
\omega=\frac{1}{n} \sum_{j=1}^{m} \int^{q_{j}} \frac{d \lambda}{\sqrt{k_{j}^{2} \lambda^{2}+b_{j} \lambda+c_{j}}}=\sum_{j=1}^{m} \ln \left(p_{j}+\frac{k_{j}^{2} q_{j}+b_{j}}{k_{j}}\right)^{\frac{1}{n k_{j}}} .
$$

So, we can easily get polynomial or rational function

$$
Z=e^{z \omega}=\prod_{j=1}^{m}\left(p_{j}+\frac{k_{j}^{2} q_{j}+b_{j}}{k_{j}}\right)^{\frac{z}{n k_{j}}}
$$

at the special choice of $k_{j}$ and $z$. In the second case $m-1$ angle variables $\omega_{k}, \ldots, \omega_{k+m-1}$ with $1<m \leq n$ have to satisfy to the Abel equations

$$
\frac{d \mathrm{x}_{1}}{\sqrt{f\left(\mathrm{x}_{1}\right)}}+\frac{d \mathrm{x}_{2}}{\sqrt{f\left(\mathrm{x}_{2}\right)}}+\cdots+\frac{d \mathrm{x}_{m}}{\sqrt{f\left(\mathrm{x}_{m}\right)}}=d \omega_{k}
$$




$$
\begin{aligned}
& \frac{\mathrm{x}_{1} d \mathrm{x}_{1}}{\sqrt{f\left(\mathrm{x}_{1}\right)}}+\frac{\mathrm{x}_{2} d \mathrm{x}_{2}}{\sqrt{f\left(\mathrm{x}_{2}\right)}}+\cdots+\frac{\mathrm{x}_{m} d \mathrm{x}_{m}}{\sqrt{f\left(\mathrm{x}_{m}\right)}}=d \omega_{k+1}, \\
& \frac{\mathrm{x}_{1}^{m-2} d \mathrm{x}_{1}}{\sqrt{f\left(\mathrm{x}_{1}\right)}}+\frac{\mathrm{x}_{2}^{m-2} d \mathrm{x}_{2}}{\sqrt{f\left(\mathrm{x}_{2}\right)}}+\cdots+\frac{\mathrm{x}_{m}^{m-2} d \mathrm{x}_{m}}{\sqrt{f\left(\mathrm{x}_{m}\right)}}=d \omega_{k+m-1}
\end{aligned}
$$

with a common polynomial of fixed degree $2 m$

$$
f(\mathrm{x}) \equiv A_{2 m} \mathrm{x}^{2 m}+A_{2 m-1} \mathrm{x}^{2 m-1}+\cdots+A_{1} \mathrm{x}+A_{0} .
$$

If this case there are some additional Richelot integrals of motion [10]

$$
C_{k}=\frac{\left[\frac{\sqrt{f\left(\mathrm{x}_{1}\right)}}{F^{\prime}\left(\mathrm{x}_{1}\right)} \cdot \frac{1}{a_{k}-\mathrm{x}_{1}}+\cdots+\frac{\sqrt{f\left(\mathrm{x}_{m}\right)}}{F^{\prime}\left(\mathrm{x}_{m}\right)} \cdot \frac{1}{a_{k}-\mathrm{x}_{m}}\right]^{2}}{\left[\frac{\sqrt{f\left(\mathrm{x}_{1}\right)}}{F^{\prime}\left(\mathrm{x}_{1}\right)}+\cdots+\frac{\sqrt{f\left(\mathrm{x}_{m}\right)}}{F^{\prime}\left(\mathrm{x}_{m}\right)}\right]^{2}-A_{2 m}} F\left(a_{k}\right) .
$$

Here $a_{k}$ are values of $\mathrm{x}$ at the branch points of the corresponding hyperelliptic curve and $F(\mathrm{x})=$ $\left(\mathrm{x}-\mathrm{x}_{1}\right)\left(\mathrm{x}-\mathrm{x}_{2}\right) \cdots\left(\mathrm{x}-\mathrm{x}_{m}\right)[10]$.

At $m=2$ we have famous Euler algebraic integral [3]. If $A_{2 m}=0$ and $A_{2 m-1} \neq 0$ in (2.3) there is another additional Richelot integrals of motion [10]. The Weierstrass generating function of such integrals for any values of the coefficients $A_{k}$ and other constructions of additional integrals of the Abel equations are discussed in [1]. Some example of the Euler and Richelot superintegrable systems may be found in $[4,12,15]$.

Below we show how these addition theorems could help us to classify superintegrable systems.

\section{Elliptic coordinate system}

Let us consider elliptic coordinates on the plane $q_{1,2}$ defined by

$$
1-\frac{x^{2}}{\lambda-\kappa}-\frac{y^{2}}{\lambda+\kappa}=\frac{\left(\lambda-q_{1}\right)\left(\lambda-q_{2}\right)}{\lambda^{2}-\kappa^{2}}, \quad \kappa \in \mathbb{R} .
$$

The corresponding momenta reads as

$$
p_{1}=\frac{2 p_{x} x}{q_{1}-\kappa}+\frac{2 p_{y} y}{q_{1}+\kappa}, \quad p_{2}=\frac{2 p_{x} x}{q_{2}-\kappa}+\frac{2 p_{y} y}{q_{2}+\kappa} .
$$

The Stäckel matrix and the separated relations

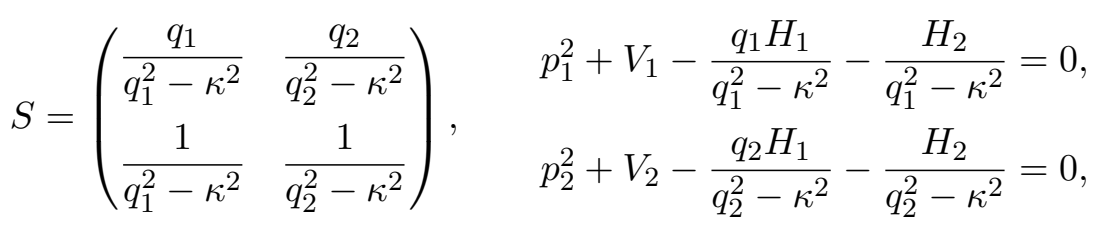

give rise to the following Hamiltonians in the involution

$$
\begin{aligned}
& H_{1}=\frac{\left(q_{1}^{2}-\kappa^{2}\right)\left(p_{1}^{2}+V_{1}\right)}{q_{2}-q_{1}}+\frac{\left(q_{2}^{2}-\kappa^{2}\right)\left(p_{2}^{2}+V_{2}\right)}{q_{2}-q_{1}}, \\
& H_{2}=\frac{q_{2}\left(q_{1}^{2}-\kappa^{2}\right)\left(p_{1}^{2}+V_{1}\right)}{q_{1}-q_{2}}-\frac{q_{1}\left(q_{2}^{2}-\kappa^{2}\right)\left(p_{2}^{2}+V_{2}\right)}{q_{1}-q_{2}} .
\end{aligned}
$$


The Hamiltonian $H_{1}$ commutes with the second angle variable $w_{2}$, which is equal to

$$
\begin{aligned}
w_{2}= & \frac{1}{2} \int^{q_{1}} \frac{d \lambda}{\sqrt{\left(\lambda^{2}-\kappa^{2}\right)\left(\lambda H_{1}+H_{2}-V_{1} \lambda^{2}+V_{1} \kappa^{2}\right)}} \\
& +\frac{1}{2} \int^{q_{2}} \frac{d \lambda}{\sqrt{\left(\lambda^{2}-\kappa^{2}\right)\left(\lambda H_{1}+H_{2}-V_{2} \lambda^{2}+V_{2} \kappa^{2}\right)}} .
\end{aligned}
$$

Polynomials

$$
P_{1,2}=\left(\lambda^{2}-\kappa^{2}\right)\left(\lambda H_{1}+H_{2}-V_{1,2} \lambda^{2}+V_{1,2} \kappa^{2}\right)
$$

standing under square root in these integrals are at least third-order polynomials on $\lambda$. So, in this case we can not apply addition theorem for the logarithms.

It is easy to see that we can apply addition theorem for the elliptic functions at

$$
V_{1}=V_{1}=\alpha .
$$

Namely, if we put $\lambda=\mathrm{x}$ and $\lambda=\mathrm{y}$ in the first and second integrals (3.1), we could apply the Euler addition theorem

$$
\frac{\mathrm{dx}}{\sqrt{\mathrm{X}}}+\frac{\mathrm{dy}}{\sqrt{\mathrm{Y}}}=\frac{\mathrm{ds}}{\sqrt{\mathrm{S}}}
$$

to angle variable $\omega_{2}$. Here $\mathrm{X}$ is an arbitrary quartic

$$
\mathrm{X}=a \mathrm{x}^{4}+4 b \mathrm{x}^{3}+6 c \mathrm{x}^{2}+4 d \mathrm{x}+e
$$

and $\mathrm{Y}, \mathrm{S}$ are the same functions of another variables $\mathrm{y}$ and $\mathrm{s}$. In this case, symmetrical biquadratic form of $\mathrm{x}$ and $\mathrm{y}$

$$
F(\mathrm{x}, \mathrm{y})=a \mathrm{x}^{2} \mathrm{y}^{2}+2 b \mathrm{xy}(\mathrm{x}+\mathrm{y})+c\left(\mathrm{x}^{2}+4 \mathrm{xy}+\mathrm{y}^{2}\right)+2 d(\mathrm{x}+\mathrm{y})+e=0
$$

defines the conic section on the plane $(\mathrm{x}, \mathrm{y})$. According to $[1,3,10]$, there is a famous Euler integral

$$
C=\frac{F(\mathrm{x}, \mathrm{y})-\sqrt{\mathrm{X}} \sqrt{\mathrm{Y}}}{2(\mathrm{x}-\mathrm{y})^{2}}=\frac{1}{4}\left(\frac{\sqrt{\mathrm{X}}-\sqrt{\mathrm{Y}}}{\mathrm{x}-\mathrm{y}}\right)^{2}-\frac{a(\mathrm{x}+\mathrm{y})^{2}}{4}-b(\mathrm{x}+\mathrm{y})-c .
$$

For the quartic (3.2) associated with the angle variable (3.1) this Euler integral looks like

$$
H_{3}=\frac{\left(p_{1}-p_{2}\right)\left(q_{1}^{2}-\kappa^{2}\right)\left(q_{2}^{2}-\kappa^{2}\right)}{\left(q_{1}-q_{2}\right)^{3}}\left(\alpha\left(q_{1}-q_{2}\right)^{2}-\left(p_{1}-p_{2}\right)^{2} \kappa^{2}+\left(p_{1} q_{1}-p_{2} q_{2}\right)^{2}\right) .
$$

Here $q_{1,2}$ and $p_{1,2}$ are elliptic coordinates and momenta.

It is a third-order polynomial in momenta which commutes with the Hamiltonian

$$
\left\{H_{1}, H_{2}\right\}=0, \quad\left\{H_{2}, H_{3}\right\}=H_{4} \neq 0 .
$$

The algebra of the polynomial integrals of motion $H_{1}, H_{2}, H_{3}$ can be closed only after some other polynomial generators are added.

Thus, we easily find the additional integrals of motion for the Hamilton function of the oscillator

$$
4 H_{1}=p_{x}^{2}+p_{y}^{2}+a\left(x^{2}+y^{2}\right)
$$

using the separation of variables in elliptic coordinate system and the corresponding angle variables. Another result is that there is only one superintegrable system separable in elliptic coordinates and associated with the known addition theorems for Abelian integrals. 


\section{Parabolic coordinate system}

Let us consider parabolic coordinates on the plane $q_{1,2}$ defined by

$$
x=q_{1} q_{2}, \quad y=\frac{q_{1}^{2}-q_{2}^{2}}{2}
$$

and the corresponding momenta

$$
p_{x}=\frac{p_{1} q_{2}+p_{2} q_{1}}{q_{1}^{2}+q_{2}^{2}}, \quad p_{y}=\frac{q_{1} p_{1}-q_{2} p_{2}}{q_{1}^{2}+q_{2}^{2}},
$$

The Stäckel matrix and the separated relations

$$
S=\left(\begin{array}{cc}
q_{1}^{2} & q_{2}^{2} \\
1 & -1
\end{array}\right), \quad \begin{aligned}
& p_{1}^{2}+V_{1}\left(q_{1}\right)-q_{1}^{2} H_{1}-H_{2}=0 \\
& p_{2}^{2}+V_{2}\left(q_{2}\right)-q_{2}^{2} H_{1}+H_{2}=0
\end{aligned}
$$

give rise to the Hamiltonians

$$
H_{1}=\frac{p_{1}^{2}+p_{2}^{+} V_{1}\left(q_{1}\right)+V_{2}\left(q_{2}\right)}{q_{1}^{2}+q_{2}^{2}}, \quad H_{2}=\frac{p_{1}^{2} q_{2}^{2}-p_{2}^{2} q_{1}^{2}+q_{2}^{2} V_{1}\left(q_{1}\right)-q_{1}^{2} V_{2}\left(q_{2}\right)}{q_{1}^{2}+q_{2}^{2}} .
$$

The Hamiltonian $H_{1}$ commutes with the second angle variable $w_{2}$, which is equal to

$$
w_{2}=\frac{1}{2} \int^{q_{1}} \frac{d \lambda}{\sqrt{\lambda^{2} H_{1}+H_{2}-V_{1}(\lambda)}}+\frac{1}{2} \int^{q_{2}} \frac{d \lambda}{\sqrt{\lambda^{2} H_{1}-H_{2}-V_{2}(\lambda)}} .
$$

In contrast with the elliptic coordinates, polynomials

$$
P_{1,2}=\lambda^{2} H_{1}+H_{2}-V_{1,2}(\lambda)
$$

standing under square root in these integrals are at least second-order polynomials on $\lambda$. So, we can apply both known addition theorems to these Abelian integrals.

In fact, these integrals are expressed via logarithmic functions iff:

Case 1: $\quad V_{1}=b_{1} q_{1}+c_{1}, \quad V_{2}=b_{2} q_{2}+c_{2}$,

Case 2: $\quad V_{1}=a_{1} q_{1}^{-2}+b_{1}, \quad V_{2}=a_{2} q_{2}^{-2}+b_{1}$.

The addition theorem for the elliptic function is applicable iff:

$$
\text { Case 3: } \quad V_{1}=a_{1} q_{1}^{6}+b_{1} q_{1}^{4}+c_{1} q_{1}^{-2}, \quad V_{2}=a_{1} q_{2}^{6}-b_{1} q_{2}^{4}+c_{1} q_{2}^{-2} .
$$

The corresponding Hamilton functions are deformations of the Kepler-Coulomb and oscillator Hamiltonians:

Case 1: $\quad H_{1}=p_{x}^{2}+p_{y}^{2}+\frac{1}{2 \sqrt{x^{2}+y^{2}}}\left(b_{1} \sqrt{x+\sqrt{x^{2}+y^{2}}}+b_{2} \sqrt{\sqrt{x^{2}+y^{2}}-x}+c_{1}+c_{2}\right)$

$$
=p_{x}^{2}+p_{y}^{2}+\frac{1}{2 r}\left(b_{1} \sqrt{2} \cos \frac{\varphi}{2}+b_{2} \sqrt{2} \sin \frac{\varphi}{2}+c_{1}+c_{2}\right) \text {; }
$$

Case 2: $\quad H_{1}=p_{x}^{2}+p_{y}^{2}+\frac{1}{2 \sqrt{x^{2}+y^{2}}}\left(\frac{a_{1}}{x+\sqrt{x^{2}+y^{2}}}+\frac{a_{2}}{x-\sqrt{x^{2}+y^{2}}}+b_{1}+b_{2}\right)$

$$
=p_{x}^{2}+p_{y}^{2}+\frac{a_{1}}{2 r^{2}(\cos \varphi+1)}-\frac{a_{2}}{2 r^{2}(\cos \varphi-1)}+\frac{b_{1}+b_{2}}{2 r} \text {; }
$$

Case 3: $\quad H_{1}=p_{x}^{2}+p_{y}^{2}+\alpha\left(4 x^{2}+y^{2}\right)+2 \beta x+\frac{\gamma}{y^{2}}$.

Here $r=\sqrt{x^{2}+y^{2}}$ and $\varphi=\arctan x / y$ are polar coordinates on the plane. According to [8] these systems remain superintegrable in the quantum case. 


\subsection{Case 1}

In the first case the second angle variable equals

$$
\begin{aligned}
\omega_{2} & =\frac{1}{2} \int^{q_{1}} \frac{d \lambda}{\sqrt{\lambda^{2} H_{1}-b_{1} \lambda+H_{2}-c_{1}}}-\frac{1}{2} \int^{q_{2}} \frac{d \lambda}{\sqrt{\lambda^{2} H_{1}-b_{2} \lambda-H_{2}-c_{2}}} \\
& =\frac{\ln \left(p_{1}-\frac{q_{1} H_{1}-b_{1} / 2}{\sqrt{H_{1}}}\right)}{2 \sqrt{H_{1}}}-\frac{\ln \left(p_{2}-\frac{q_{2} H_{1}-b_{2} / 2}{\sqrt{H_{1}}}\right)}{2 \sqrt{H_{1}}} .
\end{aligned}
$$

The application of the addition theorem (2.2) to $\omega_{2}$ gives rise to the following rational integral of motion

$$
Z=e^{2 \sqrt{H_{1}} \omega_{2}}=\frac{2 q_{1} H_{1}+2 p_{1} \sqrt{H_{1}}-b_{1}}{2 q_{2} H_{1}+2 p_{2} \sqrt{H_{1}}-b_{2}} .
$$

In order to calculate polynomial integral of motion let us consider a series expansion of the function

$$
f=\frac{1}{\sqrt{H_{1}}}\left(\alpha Z+\beta Z^{-1}\right)=\frac{\alpha\left(4 H_{1} H_{2}-4 H_{1} c_{1}-b_{1}^{2}\right)-\beta\left(4 H_{1} H_{2}+4 H_{1} c_{2}+b_{2}^{2}\right)}{\sqrt{H_{1}\left(4 H_{1} c_{2}+4 H_{1} H_{2}+b_{2}^{2}\right)\left(b_{1}^{2}+4 H_{1} c_{1}-4 H_{1} H_{2}\right)}}+O\left(p_{1,2}\right),
$$

by momenta $p_{1,2}$. Here we substitute the variables $q_{1,2}$ from the separated relations (4.1) into the rational integral $Z$ and $\alpha, \beta$ are undefined polynomials in $H_{1,2}$.

Equating first coefficient of this expansion to zero one gets the following expressions for these polynomials

$$
\alpha=4 H_{1} H_{2}+4 H_{1} c_{2}+b_{2}^{2}, \quad \beta=4 H_{1} H_{2}-4 H_{1} c_{1}-b_{1}^{2} .
$$

At this values of $\alpha$ and $\beta$ the function $f$ becomes a third-order polynomial in momenta

$$
\begin{aligned}
H_{3}= & \frac{1}{\sqrt{H_{1}}}\left(\alpha Z+\beta Z^{-1}\right)=\frac{1}{\sqrt{H_{1}}}\left(\alpha e^{2 \sqrt{H_{1}} \omega_{2}}+\beta e^{-2 \sqrt{H_{1}} \omega_{2}}\right) \\
= & \frac{8\left(p_{1} q_{2}-p_{2} q_{1}\right)\left(p_{1}^{2}+p_{2}^{2}+c_{1}+c_{2}\right)}{q_{1}^{2}+q_{2}^{2}}+\frac{4\left(2 p_{1} q_{1} q_{2}-p_{2}\left(q_{1}^{2}-q_{2}^{2}\right)\right) b_{1}}{q_{1}^{2}+q_{2}^{2}} \\
& -\frac{4\left(2 p_{2} q_{1} q_{2}+p_{1}\left(q_{1}^{2}-q_{2}^{2}\right)\right) b_{2}}{q_{1}^{2}+q_{2}^{2}},
\end{aligned}
$$

such that

$$
\left\{H_{1}, H_{3}\right\}=0 \text {. }
$$

In order to close the algebra of the polynomial integrals of motion $H_{1}, H_{2}, H_{3}$ we have to add one more polynomial generator

$$
H_{4}=\left\{H_{2}, H_{3}\right\}=2 \alpha e^{2 \sqrt{H_{1}} \omega_{2}}-2 \beta e^{-2 \sqrt{H_{1}} \omega_{2}} .
$$

by analogy with $\exp (\omega), \sin (\omega)$ and $\cos (\omega)$ functions.

Remark 1. One of the referees proposed another construction of the polynomial integrals of motion from the angle variable $\omega_{2}$. Namely, from the separation equations we can deduce that

$$
\begin{aligned}
& \Delta_{1}=\left(2 q_{1} H_{1}+2 p_{1} \sqrt{H_{1}}-b_{1}\right)\left(2 q_{1} H_{1}-2 p_{1} \sqrt{H_{1}}-b_{1}\right)=-4 H_{1} H_{2}+4 H_{1} c_{1}+b_{1}^{2}, \\
& \Delta_{2}=\left(2 q_{2} H_{1}+2 p_{2} \sqrt{H_{1}}-b_{2}\right)\left(2 q_{2} H_{1}-2 p_{2} \sqrt{H_{1}}-b_{2}\right)=4 H_{1} H_{2}+4 H_{1} c_{2}+b_{2}^{2} .
\end{aligned}
$$


We can therefore write

$$
\begin{aligned}
\Psi_{1}=\Delta_{1} Z^{-1}= & \left(\left(2 q_{1} H_{1}-b_{1}\right)\left(2 q_{2} H_{2}-b_{2}\right)-4 H_{1} p_{1} p_{2}\right) \\
& -2 \sqrt{H_{1}}\left(\left(2 q_{2} H_{1}-b_{2}\right) p_{1}-\left(2 q_{1} H_{1}-b_{1}\right) p_{2}\right)
\end{aligned}
$$

and

$$
\begin{aligned}
\Psi_{2}=\Delta_{2} Z= & \left(\left(2 q_{1} H_{1}-b_{1}\right)\left(2 q_{2} H_{2}-b_{2}\right)-4 H_{1} p_{1} p_{2}\right) \\
& +2 \sqrt{H_{1}}\left(\left(2 q_{2} H_{1}-b_{2}\right) p_{1}-\left(2 q_{1} H_{1}-b_{1}\right) p_{2}\right) .
\end{aligned}
$$

Consequently

$$
H_{3}=\frac{1}{\sqrt{H_{1}}}\left(\Psi_{1}-\Psi_{2}\right)=-4\left(\left(2 q_{2} H_{1}-b_{2}\right) p_{1}-\left(2 q_{1} H_{1}-b_{1}\right) p_{2}\right)
$$

is a third-order constant of motion. This method of explanation may be clearer than the method

of expansion of the rational in momenta function $f=\alpha Z+\beta Z^{-1}$ with indefinite coefficients $\alpha$ and $\beta$.

Remark 2. Let us remind, that two-dimensional open Toda lattice defined by the following polynomial integrals of motion

$$
H_{1}=p_{1}^{2}+p_{2}^{2}+\mathrm{e}^{q_{1}-q_{2}}, \quad H_{2}=p_{1}+p_{2},
$$

has the non-rational in momenta additional integral of motion

$$
Z=\frac{p_{1}-p_{2}+\sqrt{J}}{p_{1}-p_{2}-\sqrt{J}} \exp \left(\sqrt{J} \frac{q_{1}+q_{2}}{p_{1}+p_{2}}\right), \quad J=2 H_{2}-H_{1}^{2} .
$$

which can be also obtained from the second angle variable $\omega_{2}$ [14]. However, it is easy to prove, that we can not apply working above constructions of polynomial integral of motion $\mathrm{H}_{3}$ in this case.

\subsection{Case 2}

In the second case the angle variable is equal to

$$
\omega_{2}=\frac{1}{2} \int^{q_{1}} \frac{\lambda d \lambda}{\sqrt{\lambda^{4} H_{1}+\left(H_{2}-b_{1}\right) \lambda^{2}-a_{1}}}-\frac{1}{2} \int^{q_{2}} \frac{\lambda d \lambda}{\sqrt{\lambda^{4} H_{1}-\left(H_{2}+b_{2}\right) \lambda^{2}-a_{2}}} .
$$

Changing variables $\mu=\lambda^{2}$ one gets second-order polynomials

$$
P_{j}=\mu^{2} H_{1} \pm\left(H_{2} \mp b_{j}\right) \mu-a_{j}
$$

under the square root and desired sum of the logarithms

$$
\omega_{2}=\frac{\left.\ln \left(2 q_{1}^{2} H_{1}+2 q_{1} p_{1} \sqrt{H_{1}}+H_{2}-b_{1}\right)\right)}{4 \sqrt{H_{1}}}-\frac{\left.\ln \left(2 q_{2}^{2} H_{1}+2 q_{2} p_{2} \sqrt{H_{1}}-H_{2}-b_{2}\right)\right)}{4 \sqrt{H_{1}}} .
$$

The rational integral of motion (2.2) is equal to

$$
Z=e^{4 \sqrt{H_{1}} \omega_{2}}=\frac{2 q_{1}^{2} H_{1}+2 q_{1} p_{1} \sqrt{H_{1}}+H_{2}-b_{1}}{2 q_{2}^{2} H_{1}+2 q_{2} p_{2} \sqrt{H_{1}}-H_{2}-b_{2}} .
$$


As above we consider the expansion of the function

$$
f=\frac{1}{\sqrt{H_{1}}}\left(\alpha Z+\beta Z^{-1}\right)
$$

by momenta $p_{1,2}$. Equating first coefficient of this expansion to zero one gets polynomials $\alpha, \beta$

$$
\alpha=4 H_{1} a_{2}+b_{2}^{2}+2 b_{2} H_{2}+H_{2}^{2}, \quad \beta=-4 H_{1} a_{1}-b_{1}^{2}+2 b_{1} H_{2}-H_{2}^{2} .
$$

At this values of $\alpha$ and $\beta$ the function $f$ becomes a third-order polynomial in momenta

$$
\begin{aligned}
H_{3}= & \frac{1}{\sqrt{H_{1}}}\left(\alpha Z+\beta Z^{-1}\right)=\frac{1}{\sqrt{H_{1}}}\left(\alpha e^{4 \sqrt{H_{1}} \omega_{2}}+\beta e^{-4 \sqrt{H_{1}} \omega_{2}}\right) \\
= & \frac{4\left(q_{1} p_{1}-q_{2} p_{2}\right)\left(q_{2} p_{1}-q_{1} p_{2}\right)^{2}}{q_{1}^{2}+q_{2}^{2}}+\frac{4 q_{1} q_{2}\left(q_{2} p_{1}-q_{1} p_{2}\right)\left(b_{1}+b_{2}\right)}{q_{1}^{2}+q_{2}^{2}} \\
& +\frac{4 a_{1} q_{2}\left(q_{2} q_{1} p_{1}-\left(2 q_{1}^{2}+q_{2}^{2}\right) p_{2}\right)}{q_{1}^{2}\left(q_{1}^{2}+q_{2}^{2}\right)}-\frac{4 a_{2} q_{1}\left(q_{2} q_{1} p_{2}-\left(2 q_{2}^{2}+q_{1}^{2}\right) p_{1}\right)}{q_{2}^{2}\left(q_{1}^{2}+q_{2}^{2}\right)} .
\end{aligned}
$$

such that

$$
\left\{H_{1}, H_{3}\right\}=0 \text {. }
$$

In order to close the algebra of the polynomial integrals of motion $H_{1}, H_{2}, H_{3}$ we have to add one more polynomial generator

$$
H_{4}=\left\{H_{2}, H_{3}\right\}=4 \alpha e^{4 \sqrt{H_{1}} \omega_{2}}-4 \beta e^{-4 \sqrt{H_{1}} \omega_{2}} .
$$

\subsection{Case 3}

In the third case the angle variable is equal to

$$
\omega_{2}=\int^{q_{1}} \frac{\lambda d \lambda}{\sqrt{-a_{1} \lambda^{8}-b_{1} \lambda^{6}+H_{1} \lambda^{4}+H_{2} \lambda^{2}-c_{1}}}-\int^{q_{2}} \frac{\lambda d \lambda}{\sqrt{-a_{1} \lambda^{8}+b_{1} \lambda^{6}+H_{1} \lambda^{4}-H_{2} \lambda^{2}-c_{1}}} .
$$

Changing variables $\lambda=\sqrt{\mathrm{x}}$ and $\lambda=i \sqrt{\mathrm{y}}$ at the first and second integral one gets the Euler addition theorem (3.3). In fact, this example has been considered in Euler's book [3] too.

Identifying quartic

$$
P=-a_{1} \mu^{4}-b_{1} \mu^{3}+H_{1} \mu^{2}+H_{2} \mu-c_{1}
$$

with X (3.4) we can easily calculate the Euler integral of motion (3.5) in parabolic coordinates

$$
\begin{aligned}
H_{3}=s= & \frac{\left(q_{1} p_{1}-q_{2} p_{2}\right)\left(q_{1} p_{2}+q_{2} p_{1}\right)^{2}}{\left(q_{1}^{2}+q_{2}^{2}\right)^{3}}+\frac{a_{1} q_{1} q_{2}\left(2 q_{1}^{3} p_{2}+q_{2} q_{1}^{2} p_{1}-q_{1} q_{2}^{2} p_{2}-2 q_{2}^{3} p_{1}\right)}{q_{1}^{2}+q_{2}^{2}} \\
& +\frac{b_{1} q_{1} q_{2}\left(q_{1} p_{2}+q_{2} p_{1}\right)}{q_{1}^{2}+q_{2}^{2}}+\frac{c_{1}\left(q_{1} p_{1}-q_{2} p_{2}\right)}{q_{1}^{2} q_{2}^{2}\left(q_{1}^{2}+q_{2}^{2}\right)} .
\end{aligned}
$$

The algebra of the integrals of motion $H_{1}, H_{2}, H_{3}$ is more complicated then the algebra associated with the addition theorem for logarithms. In fact, in order to close this algebra we have to introduce the counterparts of the Jacobi elliptic functions $\operatorname{sn}(\omega), \operatorname{cn}(\omega)$ and $\operatorname{dn}(\omega)$ instead of the trigonometric functions $\sin (\omega)$ and $\cos (\omega)$, which we used for the superintegrable systems associated with the addition theorem for logarithms. 


\section{Conclusion}

It is known that orthogonal coordinate systems on Riemaniann manifolds can be viewed as an orthogonal sum of certain basic coordinate systems and these basic systems can be obtained from the elliptic coordinate system [5] using a degeneration procedure. This degeneration decreases the degree of polynomials standing under square roots into the angle variables (2.1). Thus, we have only one superintegrable systems separable in elliptic coordinates, whereas for degenerations we have a lot of different superintegrable systems. As usual, the addition theorem for logarithms allows us to get additional integrals of higher order in momenta [12, 13].

\section{Acknowledgments}

We are greatly indebted referees for several improvements and corrections induced by their comments.

\section{References}

[1] Baker H.F., Abel's theorem and the allied theory including the theory of the theta functions, Cambridge University Press, Cambridge, 1897.

[2] Borisov A.V., Kilin A.A., Mamaev I.S., Superintegrable system on a sphere with the integral of higher degree, Regul. Chaotic Dyn. 14 (2009), 615-620.

[3] Euler L., Institutiones Calculi integralis, Acta Petropolitana, 1761.

[4] Grigoryev Y.A., Khudobakhshov V.A., Tsiganov A.V., On Euler superintegrable systems, J. Phys. A: Math. Theor. 42 (2009), 075202, 11 pages.

[5] Kalnins E.G., Separation of variables for Riemannian spaces of constant curvature, Pitman Monographs and Surveys in Pure and Applied Mathematics, Vol. 28, Longman Scientific \& Technical, Harlow, 1986.

[6] Kalnins E.G., Miller W., Structure theory for extended Kepler-Coulomb 3D classical superintegrable systems, arXiv:1202.0197.

[7] Maciejewski A.J., Przybylska M., Tsiganov A.V., On algebraic construction of certain integrable and superintegrable systems, Phys. D 240 (2011), 1426-1448, arXiv:1011.3249.

[8] Popperi I., Post S., Winternitz P., Third-order superintegrable systems separable in parabolic coordinates, arXiv:1204.0700.

[9] Post S., Winternitz P., A nonseparable quantum superintegrable system in 2D real Euclidean space, J. Phys. A: Math. Theor. 44 (2011), 162001, 8 pages, arXiv:1101.5405.

[10] Richelot F., Ueber die Integration eines Merkwürdigen Systems von Differentialgleichungen, J. Reine Angew. Math. 23 (1842), 354-369.

[11] Stäckel P., Über die Integration der Hamilton-Jacobischen Differential Gleichung Mittelst Separation der Variabeln, Habilitationsschrift, Halle, 1891.

[12] Tsiganov A.V., Addition theorems and the Drach superintegrable systems, J. Phys. A: Math. Theor. 41 (2008), 335204, 16 pages, arXiv:0805.3443.

[13] Tsiganov A.V., Leonard Euler: addition theorems and superintegrable systems, Regul. Chaotic Dyn. 14 (2009), 389-406, arXiv:0810.1100.

[14] Tsiganov A.V., On maximally superintegrable systems, Regul. Chaotic Dyn. 13 (2008), 178-190, arXiv:0711.2225.

[15] Tsiganov A.V., On the superintegrable Richelot systems, J. Phys. A: Math. Theor. 43 (2010), 055201, 14 pages, arXiv:0909.2923. 